\title{
Helicobacter pylori infection, but not genetic polymorphism of CYP2E1, is highly prevalent in gastric cancer patients younger than 40 years
}

\author{
Gotaro Masuda $^{1}$, Akira Tokunaga ${ }^{2}$, Takashi Shirakawa ${ }^{3}$, Akiyoshi Togashi ${ }^{1}$, Teruo Kiyama ${ }^{1}$, Shunj Kato $^{1}$, \\ Norio Matsukura ${ }^{1}$, Hideki Bou ${ }^{2}$, Masanori Watanabe ${ }^{2}$, and TAKashi TaJiri ${ }^{1}$ \\ ${ }^{1}$ Surgery for Organ and Biological Regulation (First Department of Surgery), Nippon Medical School, Tokyo, Japan \\ ${ }^{2}$ Institute of Gastroenterology, Nippon Medical School Musashikosugi Hospital, 1-396 Kosugicho, Nakahara-ku, Kawasaki, \\ Kanagawa 211-8533, Japan \\ ${ }^{3}$ Department of Surgery, Nippon Medical School Chiba Hokusoh Hospital, Chiba, Japan
}

\begin{abstract}
Background. Gastric cancers in young adults are thought to be associated with risk factors that include Helicobacter pylori infection and genetic polymorphism. The objective of this study was to elucidate the roles of these risk factors in patients younger than $\mathbf{4 0}$ years by analyzing clinicopathological data and $\boldsymbol{H}$. pylori infection, and using molecular epidemiologic techniques.

Methods. Clinicopathological features, the presence of $\boldsymbol{H}$. pylori infection, endoscopic characteristics of gastritis, genetic polymorphism of P4502E1 (CYP2E1), and family history of cancer in patients with gastric cancer treated surgically at Nippon Medical School Hospital from 1991 to 2004 were analyzed, based on our medical database.

Results. Gastric cancer in those younger than 40 years was characterized by a predominance of female patients with poorly differentiated adenocarcinoma who had undergone total gastrectomy with extended lymphadenectomy. $\mathrm{H}$. pylori infection had a higher prevalence in patients with gastric cancer than in patients with normal endoscopic results or chronic gastritis, especially in those younger than 40 years (odds ratio, 13.7). Atrophic gastritis, nodular gastritis, and rugal hyperplastic gastritis were observed by endoscopy as $\boldsymbol{H}$. pyloriassociated gastritis. No difference in the incidence of either CYP2E1 genetic polymorphism or a family history of cancer was observed among different age groups.

Conclusion. Gastric cancer in patients younger than 40 years is closely associated with $H$. pylori infection, but not with genetic characteristics. Eradication therapy for $\boldsymbol{H}$. pylori and endoscopic examination of $\boldsymbol{H}$. pylori-positive young adults may be anticipated to be adopted as a strategy for the prevention and/or early detection of cancer.
\end{abstract}

Key words Gastric cancer - Young adults · Helicobacter pylori

Offprint requests to: A. Tokunaga

Received: September 24, 2006 / Accepted: February 25, 2007

\section{Introduction}

Genetic factors and/or exposure to environmental factors may contribute to the occurrence of human cancers. Risk factors for gastric cancer have been shown to include polymorphisms of nitroso compound activating enzyme [1,2], and the genetic polymorphisms [3-5] may be responsible for susceptibility to cancer as endogenous and/or host factors; as well, Helicobacter pylori infection [6-10], excessive sodium chloride intake [11], and Epstein-Barr (EB) virus infection [12] appear to play pivotal roles in the development of the cancer, as exogenous factors.

Reports [13-15] describing the occurrence of gastric cancer as being derived from the pathway of $H$. pyloriassociated atrophic gastritis have been published. Longterm infection with $H$. pylori has been reported to result in $H$. pylori-associated chronic atrophic gastritis, which has been shown to correlate closely with gastric cancer, especially in young adults [16-19]. The endoscopic characteristics of $H$. pylori-associated gastritis in young adults have revealed nodular, rugal hyperplastic, and chronic atrophic gastritis to be specific to patients with H. pylori infection [20].

Cytochrome P4502E1 (CYP2E1) activates carcinogenic N-nitrosamines and other potential human carcinogens. Genetic polymorphisms of CYP2E1 have been shown to confer individual susceptibility to human cancers. Esophago-gastrointestinal cancers have been reported to be relevant to the expression of CYP2E1 and to N-nitrosamine metabolism [21-23]. It would thus be worthwhile to investigate whether the frequency of CYP2E1 restriction fragment-length polymorphism (RFLP) differs among gastric cancer patients younger than 40 years, patients in their sixties, and those 80 years or older.

The present studies were therefore designed to clarify the risk factors for gastric cancer in young adults; 
the clinicopathological features of gastric cancer, based on clinical and pathological records; the incidences of $H$. pylori infection and $H$. pylori-associated gastritis, detected by blood tests and endoscopic biopsy; the frequency of CYP2E1 genetic polymorphism; and family history. Based on the results, current trials aimed at the reduction of risk factors are briefly discussed.

\section{Patients and methods}

A medical database containing retrospective documented variables in patients treated consecutively for gastric cancer at our hospital from 1991 to 2004 was analyzed. All patients had undergone gastrectomy with D0, D1, D2 or D3 type lymphadenectomy.

\section{Clinicopathological studies}

Clinicopathological findings, classified according to the Japanese Gastric Cancer Association Japanese classification of gastric carcinoma [24] and obtained from operative records and pathology reports, were filed using the File Maker Pro system (FileMaker, Santa Clara, CA, USA). The gastric cancer cases were then divided into early and advanced types according to the depth of tumor invasion in the gastric wall.

\section{Surgical techniques}

Five faculty surgeons, all specialists in gastric surgery, performed the operations. The most appropriate surgical procedure (total, proximal, or distal gastrectomy) was selected based on location, tumor size, and the extent of nodal involvement. For stage I gastric cancer surgery, the degree of lymph node dissection was selected at the surgeon's discretion. D2 dissection was the standard method for stages II and III, and D3 dissection was selected for nonelderly patients with stage III gastric cancer.

Endoscopic diagnosis of gastric cancer and $\mathrm{H}$. pyloriassociated gastritis

Endoscopic diagnosis was performed with gastrointestinal (GI) fiberscopes by a specialist in GI endoscopy. Retrospectively, the final diagnosis of gastric cancer and H. pylori-associated gastritis was made independently, based on observation of the endoscopic films by two GI endoscopy specialists. Any diagnostic discrepancy was discussed until agreement was reached. Criteria for endoscopic diagnosis were based on the Sydney system [25], and nodular gastritis was diagnosed according to previous reports [26,27].
Enzyme-linked immunosorbent assay (ELISA) for serum IgG antibody to $\mathrm{H}$. pylori in patients with gastric cancer, those with peptic ulcer, and those with chronic gastritis who were endoscopically normal

Blood samples were collected and stored at $-80^{\circ} \mathrm{C}$ until the assay. Serum IgG antibody to H. pylori was assayed by ELISA (AMRAD Operation, Australia), and absorbance at $450 \mathrm{~nm}$ was measured. The results were expressed quantitatively, with less than $30 \mathrm{U} / \mathrm{ml}$ regarded as a negative result.

Detection of $\mathrm{H}$. pylori by endoscopic biopsy in patients with gastric cancer, those with peptic ulcer, and those with chronic gastritis who were endoscopically normal

Endoscopic biopsy specimens were collected from: (1) the greater curvature of the antrum; (2) the greater curvature of the upper corpus; and (3) the lesser curvature of the lower corpus, for the detection of $\mathrm{H}$. pylori. Hematoxylin-and-eosin staining, improved toluidine blue staining, and $H$. pylori-specific-antibody immunostaining (DAKO, Glostrup, Denmark) were used for the histological detection of $H$. pylori organisms.

\section{Restricted fragment-length polymorphism (RFLP) analysis of CYP2E1}

DNA was isolated from peripheral blood buffy coats by phenol extraction methods, as previously described. The polymerase chain reaction (PCR) was used to amplify the transcription regulatory region of CYP2E1, which includes the restriction enzyme recognition site for $R s a \mathrm{I}$. Genomic DNA $(0.1 \mu \mathrm{g})$ was amplified with primers (5'-TTCATTCTGTCTTCTAACTGG and 5'TTCATTCTGTCTTCTAACTCG) according to previously reported methods [28]. The PCR product $(15 \mu \mathrm{l})$ was subjected to $R s a \mathrm{I}$ restriction enzyme analysis by electrophoresis in an agarose gel $(2.2 \%)$. The presence of the restriction site yielded two fragments, of 360 and $52 \mathrm{bp}$.

\section{Family history}

Family histories of the patients with gastric cancer were obtained by interviews conducted by attending doctors, who spoke to the patients themselves and/or their relatives; the presence of cancer in second-degree relatives was taken as a positive family history.

\section{Statistical analysis}

Statistical analysis was checked by the $\chi^{2}$ test, and cumulative survival rates were calculated using the Kaplan-Meier method and Stat View software (SAS 
Institute, USA); survival curves were tested using the Logrank method. A $P$-value of less than 0.05 was considered significant.

\section{Informed consent}

All patients were informed of their therapeutic options. No treatments were performed without informed consent.

\section{Ethics}

The study protocol conformed to the ethical guidelines established by the 1975 Declaration of Helsinki and was approved by the institutional review board of Nippon Medical School.

\section{Results}

Clinicopathological features of gastric cancer in patients younger than 40 years

Patients younger than 40 years were characterized by a prevalence of female patients, mid-stomach cancer location, and poorly differentiated adenocarcinoma histologically.

\section{Incidence of $\mathrm{H}$. pylori infection}

The H. pylori infection results in 31 patients younger than 40 years were used in this study. The positive rate in this group was $71 \%$ in males, and $88 \%$ in females. The positive rates for $H$. pylori in patients with gastric cancer regardless of age $(n=362)$ ranged from $70 \%$ to $80 \%$, and were unaffected by age. The positive rates in patients with normal endoscopic findings and chronic gastritis $(n=721)$ were $23.3 \%$ in those younger than 40 years, $48.4 \%$ in patients in their forties, and $56.8 \%$,
$61.7 \%, 60.7 \%$, and $55.6 \%$ in patients in their fifties, sixties, seventies, and those 80 years or older, respectively. In the peptic ulcer group $(n=939)$, the positive rate was more than $70 \%$ in those in their thirties to sixties, but the prevalence declined after the seventies. The odds ratios for $H$. pylori infection in patients with gastric cancer and the patients with normal endoscopic findings and chronic gastritis are shown in Table 1, the highest odds ratio was 13.69 , in patients younger than 40 years. Odds ratios were higher in patients with gastric cancer than in those with normal endoscopic findings and chronic gastritis, regardless of age.

\section{H. pylori-associated gastritis}

Fifteen of the 31 (48.4\%) patients aged less than 40 years were diagnosed as having atrophic gastritis in the gastric body; 3 of the $31(9.7 \%)$ also had nodular gastritis in the antrum, and 1 of the $31(3.2 \%)$ showed rugal hyperplastic gastritis in the gastric body.

\section{RFLP analysis of CYP2E1}

The results of the CYP2E1 polymorphism analysis in patients with gastric cancer are shown in Table 2, stratified by age group for those younger than 40 years, those in their sixties, and those 80 years or older. In the CYP2E1 RsaI restriction analysis, the homozygous minor genotype $(\mathrm{R} / \mathrm{R})$ and heterozygous genotype $(\mathrm{C} /$ $\mathrm{R})$ were not found to differ significantly among the three groups.

\section{Family history of cancer}

Table 2 also shows the prevalence of a family history of cancer, stratified by the above three age groups. No significant differences were observed among the three groups.

Table 1. Odds ratios for Helicobacter pylori infection positive rates in patients with gastric cancer versus patients with normal endoscopic findings and chronic gastritis

\begin{tabular}{llcc}
\hline & \multicolumn{2}{c}{ H. pylori infection } \\
\cline { 2 - 3 } Age (years) & Gastric cancer & $\begin{array}{c}\text { Endoscopically normal } \\
+ \text { chronic gastritis }\end{array}$ & Odds ratio (95\% CI) \\
\hline$\leq 39$ & $25 / 31(80.6 \%)$ & $28 / 120(23.3 \%)$ & $13.69(5.11-36.71)$ \\
$40-49$ & $36 / 49(73.5 \%)$ & $46 / 95(48.4 \%)$ & $2.95(1.39-6.25)$ \\
$50-59$ & $52 / 67(77.6 \%)$ & $104 / 183(56.8 \%)$ & $2.63(1.38-5.02)$ \\
$60-69$ & $94 / 128(73.4 \%)$ & $111 / 180(61.7 \%)$ & $1.72(1.05-2.82)$ \\
$70-79$ & $49 / 64(76.6 \%)$ & $65 / 107(60.7 \%)$ & $2.11(1.05-4.24)$ \\
$\geq 80$ & $16 / 23(69.6 \%)$ & $20 / 36(55.6 \%)$ & $1.83(0.61-5.52)$ \\
\hline
\end{tabular}


Table 2. Comparison of genetic characteristics of patients with gastric cancer among three groups (patients younger than 40 years, patients in their sixties, and patients 80 years or older), showing prevalence of cytochrome P4502E1 (CYP2E1) genetic polymorphism and prevalence of a family history of cancer

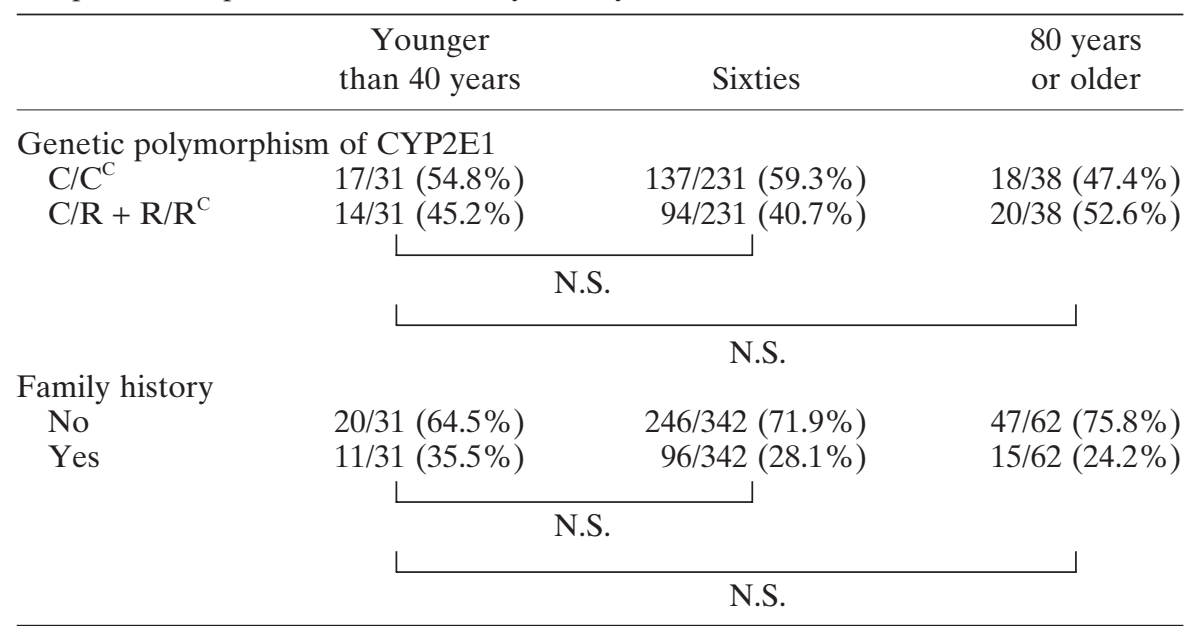

N.S., not significant

\section{Discussion}

In 31 patients younger than 40 years with gastric cancer who underwent surgery between 1991 and 2004, the clinicopathological features, presence of $H$. pylori infection, and characteristics of gastritis were examined; CYP2E1 genetic polymorphism and family history were also investigated, in comparison with findings in patients with gastric cancer in their sixties and 80 years or older.

Clinicopathological features in the 31 patients younger than 40 years with gastric cancer were characterized by the predominance of female patients with poorly differentiated adenocarcinoma, located in the middle part of the stomach, many of whom had undergone total gastrectomy with D2 or D3 lymph node dissection. The predominance of female patients with poorly differentiated adenocarcinoma that we observed was in accordance with other reports [29,30].

As compared with the results in the patients with normal endoscopic findings and chronic gastritis, the predominance of $H$. pylori infection in the patients younger than 40 was striking; $71 \%$ of the male and $88 \%$ of the female patients being positive for $\mathrm{H}$. pylori. Gastric cancer in young adults has been found to be associated with $H$. pylori infection. An increasing number of reports have stressed the association of $H$. pylori infection with the development of gastric cancer in young adults [16-18]. The question of the role of $H$. pylori infection in the gastric mucosa in the development of the diffuse type of cancer without mucosal atrophy remains open, in contrast to the role of this infection in the intestinal type of cancer with mucosal atrophy and intestinal metaplasia. The relationship between $H$.

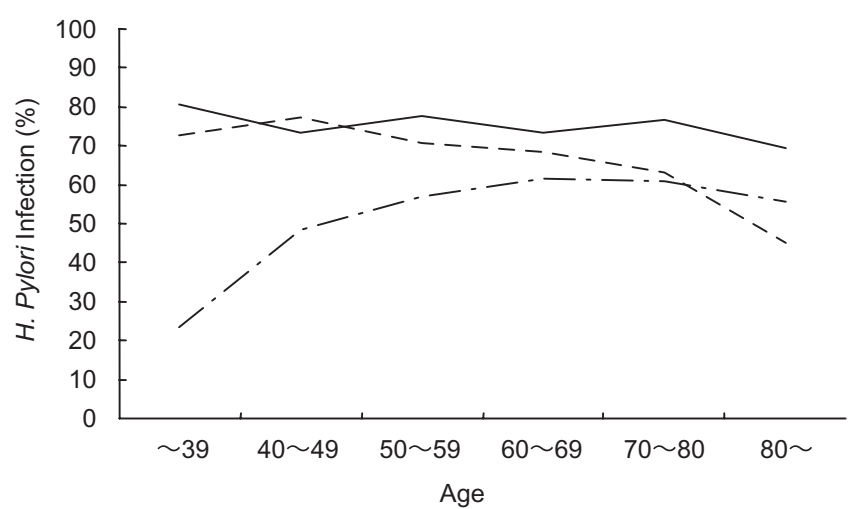

Fig. 1. Helicobacter pylori infection positive rates according to gastric disease for various age groups. Continuous line, patients with gastric cancer $(n=362)$; dashed line, patients with gastric ulcer + duodenal ulcer (peptic ulcer; $n=939$ ); dotted/dashed line, patients with normal endoscopic findings + chronic gastritis $(n=721)$

pylori-associated gastritis and the development of the diffuse type of cancer has been considered by Uemura et al. [14], who noted that gastric mucosa with severe inflammation and moderate atrophy induced by $H$. pylori infection had a potential risk for undifferentiated gastric cancer. Miyamoto et al. [26,27] have reported that nodular gastritis which is induced by $H$. pylori infection is closely associated with gastric cancer in young adults. A bird-skin appearance in endoscopy and hyperplasia of B-lymphocytes are characteristics of nodular gastritis, which is considered to represent marked inflammation. Active and chronic inflammatory cellular infiltrations were found to correlate with $H$. pylori in- 
fection in a study of gastric remnants [31]. However, there is a limit to the relevance of discussing gastric cancer development in various kinds of surgically resected specimens. H. pylori infection evidently causes inflammation of the gastric mucosa, and cytokines derived from inflammatory cells may transform epithelial or mesenchymal cells, resulting in gastric cancer or mucosa-associated lymphoid tissue (MALT) lymphoma. A recent report has reviewed the clinical outcomes of gastric lesions induced by $H$. pylori infection, which depend mainly on host factors [32].

Other recent reports have suggested that CYP2E1 genetic polymorphism is responsible for susceptibility to GI cancers as well as lung cancer [21-23]. The present study yielded negative results regarding an association of CYP2E1 genetic polymorphism with the occurrence of gastric cancer in patients younger than 40 years. No association of CYP2E1 genetic polymorphism or other genetic factors with gastric cancer has been reported previously [28].

Familial clustering of cancer in patients with gastric cancer was studied in the present investigation, but no difference in the prevalence of family history was found among the three age groups we examined. Familial aggregation was reported to be specific for the intestinal type of gastric cancer in a patient with a positive family history [33]. Intrafamilial clustering of $H$. pylori infection was reported to have a crucial role in familial aggregation of gastric cancer [34].

In conclusion, a high prevalence of $H$. pylori infection in patients with gastric cancer younger than 40 years was confirmed in the present study. Eradication therapy for $H$. pylori would therefore be expected to prevent gastric cancer development, as this cancer arises from $H$. pylori-associated gastritis. Furthermore, endoscopic GI examinations in people who are $H$. pylori-positive would promote the early detection of cancer. Trials of $H$. pylori eradication therapy for the prevention of gastric cancer are currently underway in China and Japan, and promising results are being obtained [35,36].

\section{References}

1. Yang CS, Yoo JS, Ishizaki H, Hong JY. Cytochrome P450IIE1: role in nitrosamine metabolism and mechanisms of regulation. Drug Metab Rev 1990;22:147-9.

2. Guengerich FP, Kim DH, Iwasaki M. Role of human cytochrome P-450 IIE1 in the oxidation of many low molecular weight cancer suspects. Chem Res Toxicol 1991;4:167-79.

3. Bevan S, Houlston RS. Genetic predisposition to gastric cancer. QJM 1999;92:5-10.

4. Miao X, Xing D, Tan W, Qi J, Lu W, Lin D. Susceptibility to gastric cardia adenocarcinoma and genetic polymorphysm in methylenetetrahydrofolate reductase in an at-risk Chinese population. Cancer Epidemiol Biomarkers Prev 2002;11:14548.
5. Zhu Y, Spitz MR, Amos CI, Lin MB, Schabath MB, Wu X. An evolutionary perspective on single-nucleotide polymorphism screening in molecular cancer epidemiology. Cancer Res 2004;64: 2251-7.

6. Blaser MJ, Perez-Perez GI, Kleanthous H, Cover TL, Peek RM, Chyou PH, et al. Infection with Helicobacter pylori strains possessing cag A is associated with an increased risk of developing adenocarcinoma of the stomach. Cancer Res 1995;55:2111-5.

7. Uemura N, Mukai T, Okamoto S, Yamaguchi S, Mashiba H, Taniyama K, et al. Effect of Helicobacter pylori eradication on subsequent development of cancer after endoscopic resection of early gastric cancer. Cancer Epidemiol Biomarkers Prev 1997;6: 639-42.

8. Parsonnet J, Friedman GD, Orentriech N, Vogelman H. Risk for gastric cancer in people with CagA positive or CagA negative Helicobacter pylori infection. Gut 1997;40:297-301.

9. Kikuchi S. Epidemiology of Helicobacter pylori and gastric cancer. Gastric Cancer 2002;5:6-15.

10. El-Omar EM, Rabkin CS, Gammon MD, Vaughan TL, Risch HA, Schoenberg JB, et al. Increased risk of noncardia gastric cancer associated with proinflammatory cytokine gene polymorphisms. Gastroenterology 2003;124:1193-201.

11. Shikata K, Kiyohara Y, Kubo M, Yonemoto K, Ninomiya T, Shirota $\mathrm{T}$, et al. A prospective study of dietary salt intake and gastric cancer incidence in a defined Japanese population: The Hisayama study. Int J Cancer 2006;119:196-201.

12. Burke AP, Yen TS, Shekitka KM, Sobin LH. Lymphoepithelial carcinoma of the stomach with Epstein-Barr virus demonstrated by polymerase chain reaction. Mod Pathol 1990;3:377-80.

13. Correa P. Human gastric carcinogenesis: a multistep and multifactorial process.-First American Cancer Society Award Lecture on Cancer Epidemiology and Prevention. Cancer Res 1992;52: $6735-40$.

14. Uemura N, Okamoto S, Yamamoto S, Matsumura N, Yamaguchi $\mathrm{S}$, Yamakido M, et al. Helicobacter pylori infection and the development of gastric cancer. N Engl J Med 2001;345:784-9.

15. Takahashi S. Long-term Helicobacter pylori infection and the development of atrophic gastritis and gastric cancer in Japan. J Gastroenterol 2002;37:24-7.

16. Kikuchi S, Wada O, Nakajima T, Nishi T, Kobayashi O, Konishi $\mathrm{T}$, et al. Serum anti-Helicobacter pylori antibody and gastric carcinoma among young adults. Cancer 1995;75:2789-93.

17. Kokkola A, Valle J, Haapiainen R, Sipponen P, Kivilaakso E, Puolakkainen P. Helicobacter pylori infection in young patients with gastric carcinoma. Scand J Gastroenterol 1996;31:643-7.

18. Koshida Y, Koizumi W, Sasabe M, Katoh Y, Okayasu I. Association of Helicobacter pylori-dependent gastritis with gastric carcinomas in young Japanese patients: histopathological comparison of diffuse and intestinal type cancer cases. Histopathology 2000; 37:124-30.

19. Ricuarte O, Gutierrez O, Cardona H, Kim JG, Graham DY, ElZimaity HM. Atrophic gastritis in young children and adolescents. Clin Pathol 2005;58:1189-93.

20. Shimatani T, Inoue M, Iwamoto K, Hyogo H, Yokozaki M, Saeki $\mathrm{T}$, et al. Prevalence of Helicobacter pylori infection, endoscopic gastric findings and dyspeptic symptoms among a young Japanese population born in the 1970s. J Gastroenterol Hepatol 2005;20: 1352-7.

21. Godoy W, Albano RM, Moraes EG, Alves Pinho PR, Nues RA, Saito EH, et al. CYP2A6/2A7 and CYP2E1 expression in human oesophageal mucosa; regional and inter-individual variation in expression and relevance to nitrosamine metabolism. Carcinogenesis 2002;23:611-6.

22. Marchand LL, Donlon T, Seifried A, Wilkens LR. Red meat intake, CYP2E1 polymorphisms, and colorectal cancer risk. Cancer Epidemiol Biomarkers Prev 2002;11:1019-24.

23. Cai L, Zheng ZL, Zheng ZF. Cytochrome p450 2E1 polymorphisms and the risk of gastric cardia cancer. World J Gastroenterol 2005;11:1867-71. 
24. Japanese Gastric Cancer Association. Japanese classification of gastric carcinoma. 2nd English Ed. Gastric Cancer 1998;1:10-24.

25. Tytgat GN. The Sydney System: endoscopic division. Endoscopic appearances in gastritis/duodenitis. J Gastroenterol Hepatol 1991; 6:223-34.

26. Miyamoto M, Haruma K, Yoshihara M, Hiyama T, Sumioka M, Nishisaka T, et al. Nodular gastritis in adults is caused by Helicobacter pylori infection. Dig Dis Sci 2003;48:968-75.

27. Miyamoto M, Haruma K, Yoshihara M, Sumioka M, Nishisaka $\mathrm{T}$, Tanaka S, et al. Five cases of nodular gastritis and gastric cancer: a possible association between nodular gastritis and gastric cancer. Dig Liver Dis 2002;34:819-20.

28. Kato S, Onda M, Matsukura N, Tokunaga A, Tjiri T, Kim DY et al. Cytochrome P450 (CYP2E1) genetic polymorphism in a case-control study of gastric cancer and liver disease. Pharmacogenetics 1995;5:s141-4.

29. Ramos-De la Medina A, Salgado-Nesme N, Torres-Villalobos G, Medina-Franco H. Clinicopathologic characteristics of gastric cancer in a young patient population. J Gastroint Surg 2004;8: 240-4.

30. Llanos O, Butte JM, Crovari F, Duarte I, Guzman S, Survival of young patients after gastrectomy for gastric cancer. World J Surg 2006;30:17-20.
31. Lee Y, Tokunaga A, Tajiri T, Masuda G, Okuda T, Fujita I, et al. Inflammation of the gastric remnant after gastrectomy: mucosal erythema is associated with bile reflux and inflammatory cellular infiltration is associated with Helicobacter pylori infection. J Gastroenterol 2004;39:520-6.

32. Chiba T, Seno H, Marusawa H, Wakatsuki Y, Okazaki K. Host factors are important in determining clinical outcome of Helicobacter pylori infection. J Gastroenterol 2006;41:1-9.

33. Bernini M, Barbi S, Roviello F, Scarpa A, Moore P, Pedrazzani $\mathrm{C}$, et al. Family history of gastric cancer: a correlation between epidemiologic findings and clinical data. Gastric Cancer 2006;9: 9-13.

34. Chang YW, Han YS, Lee DK, Kim HJ, Moon JS, Dong SH, et al. Role of Helicobacter pylori infection among offspring or siblings of gastric cancer patients. Int J Cancer 2002;101:46974.

35. Wong BC, Lam SK, Wong WM, Chen JS, Zheng TT, Feng RE, et al. Helicobacter pylori eradication to prevent gastric cancer in a high risk region of China: a randomized controlled trial. JAMA 2004;291:344-6.

36. Saito D. H. pylori and gastric cancer: Japanese Intervention Trial of H. pylori. In: Proc 78th Japan Gastric Cancer Assoc; 2006. p. 163. 